\title{
Systematic review of intravenous immunoglobulin in haemolytic disease of the newborn
}

\section{R Gottstein, R W I Cooke}

See end of article for authors' affiliations

......................

Correspondence to: Dr Gottstein, Neonatal Unit, Liverpool Women's Hospital, Crown Street, Liverpool L8 7SS, UK; rgottstein@btinternet.com

Accepted 23 April 2002

\begin{abstract}
Objectives: To assess the effectiveness of high dose intravenous immunoglobulin (HDIVIG) in reducing the need for exchange transfusion in neonates with proven haemolytic disease due to Rh and/or $A B O$ incompatibility. To assess the effectiveness of HDIVIG in reducing the duration of phototherapy and hospital stay.

Design: Systematic review of randomised and quasi-randomised controlled trials comparing HDIVIG and phototherapy with phototherapy alone in neonates with $\mathrm{Rh}$ and/or $\mathrm{ABO}$ incompatibility.

Results: Significantly fewer infants required exchange transfusion in the HDIVIG group (relative risk (RR) 0.28 (95\% confidence interval (Cl) 0.17 to 0.47$)$; number needed to treat $2.7(95 \% \mathrm{Cl} 2.0$ to 3.8)). Also hospital stay and duration of phototherapy were significantly reduced.

Conclusion: HDIVIG is an effective treatment.
\end{abstract}

for ntravenous immunoglobulin (IVIG) has been used widely for the treatment of immunodeficiency syndromes and several autoimmune diseases including idiopathic thrombocytopenic purpura in children ${ }^{12}$ and adults. ${ }^{3}$ It has been given to pregnant women for the treatment of idiopathic thrombocytopenic purpura ${ }^{4}$ and for the prenatal treatment of known alloimmune thrombocytopenia ${ }^{5}$ and severe erythroblastosis fetalis. ${ }^{6}$ IVIG has also been administered directly to the fetus, both in combination with and without intrauterine intravascular transfusions for severe RhD haemolytic disease..$^{78}$ In the neonatal period, IVIG has been used for the treatment of alloimmune thrombocytopenia, ${ }^{9}$ as an additional treatment in neonatal infections, ${ }^{10}$ and in the rare cases of neonatal Kawasaki disease. ${ }^{11}$ As a progression from its use for the treatment of erythroblastosis fetalis and from experience in neonates for other indications, high dose IVIG (HDIVIG) is now used in the treatment of haemolytic disease of the newborn (HDN).

Antenatal HDIVIG given to pregnant women in cases of severe rhesus D alloimmunisation has had variable success. One retrospective review showed a reduction in the percentage of severely anaemic fetuses and fetal mortality with antenatal IVIG infusion. ${ }^{6}$ Some case reports showed no apparent effect on fetal hydrops or maternal antibody titres in the treatment of Rh disease, but in one patient with Kell sensitisation the severity of the disease appeared to be ameliorated..$^{12}$ A single randomised study of low dose IVIG infusion directly into the fetal vascular system in combination with intravascular transfusion showed no beneficial effect. ${ }^{7}$ However, the numbers were small, with 10 patients in each study arm.

The exact mechanism of action of IVIG in HDN is still unknown. It is thought that the erythrocyte destruction is similar to destruction of antibody sensitised platelets in neonatal isoimmune thrombocytopenia. In the case of $\mathrm{Rh}$ haemolytic disease, for example, it is hypothesised that the anti-D sensitised neonatal erythrocytes are destroyed by antibody dependent cellular cytotoxic effects mediated by the FC receptor on the cells of the reticuloendothelial system. Haemolysis and rising bilirubin levels ensue when the Fc portion of the anti-D antibody on the surface of the neonatal erythrocytes comes into contact with the Fc receptor of the cells of the reticuloendothelial system. HDIVIG would occupy the Fc receptor sites, thus competing with the anti-D sensitised neonatal erythrocytes and preventing further haemolysis. ${ }^{13}$
In this systematic review, we evaluate the role of postnatal administration of HDIVIG to neonates with HDN.

\section{METHODS \\ Criteria for considering studies}

We identified randomised and quasi-randomised controlled trials of infants with proven $\mathrm{Rh}$ and/or ABO HDN reporting on at least one of the following outcomes: number of exchange transfusions, length of phototherapy, or length of hospital stay. The interventions under investigation were HDIVIG with phototherapy or phototherapy alone. Thus all infants received phototherapy and the only variable was the postnatal administration of HDIVIG. There were no birth weight or gestational age limits, and no limitation on severity of haemolytic disease or whether the infants or their mothers received antenatal treatment. Trials were not limited in terms of dose or duration of HDIVIG infusion.

\section{Literature search}

The literature search was performed in November 2000 using Medline (1986-2000), Embase (1984-2000), and Cochrane Library Controlled Trials Registers using the following subject and text word terms: "neonate(s), newborn(s), or infant(s)"; "rhesus incompatibility, rhesus isoimmunization, rhesus alloimmunisation, rhesus alloimmunization, ABO incompatibility, $\mathrm{ABO}$ isoimmunisation, $\mathrm{ABO}$ isoimmunization, $\mathrm{h}(\mathrm{a})$ emolytic disease, $\mathrm{h}(\mathrm{a})$ emolytic jaundice or erythroblastosis $\mathrm{f}(\mathrm{o})$ etalis"; and "immunoglobulin, immune globulin, or gammaglobulin". A manual search of the Journal of Pediatrics (1985-2000), Pediatrics (1990-2000), and Archives of Disease in Childhood (1990-2000) was conducted and included abstracts submitted to scientific meetings. Additional citations were sought using references in articles retrieved from the searches. Unpublished and further published trials were sought from experts in the field. Searches were not limited by language.

Abbreviations: IVIG, intravenous immunoglobulin; HDIVIG, high dose IVIG; HDN, haemolytic disease of the newborn; RR, relative risk; RD, risk difference; WMD, weighted mean difference; $\mathrm{Cl}$, confidence interval NNT, number needed to treat 


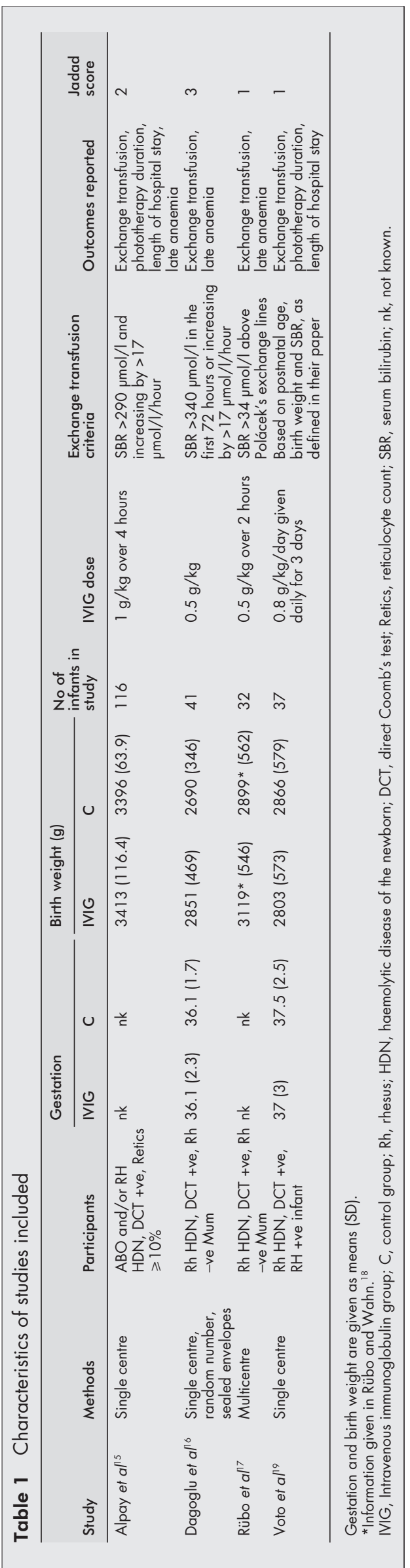

Analysis

Methodological quality was assessed using Jadad criteria. ${ }^{14}$ Outcome information on the number of infants requiring exchange transfusions, the number requiring red cell transfusions for late anaemia, as well as the total number of exchange and red cell transfusions performed was abstracted. Information on length of hospital stay and duration of phototherapy was collected. Reports of side effects or absence thereof were recorded. Where data were incomplete, the authors were contacted by letter for further information or clarification. The statistical package (RevMan version 4.1) provided by the Cochrane Collaboration was used. Relative risk (RR) and risk difference ( $R D)$ using the fixed effects model are reported for dichotomous data, and weighted mean difference (WMD) for continuous data, with their $95 \%$ confidence intervals $(\mathrm{CI})$.

\section{RESULTS}

From the literature search, six randomised controlled trials were identified as meeting the criteria for inclusion in the review. ${ }^{15-20}$ One publication ${ }^{18}$ describes a previously reported study ${ }^{17}$ and gave no additional information or data. Therefore only the initial publication was included. A further study ${ }^{20}$ investigating two dosage regimens of HDIVIG was excluded, because errors had occurred during randomisation resulting in the control group having significantly higher haemoglobin levels at entry to the trial (and thus a disease of lesser severity). The data were not analysed on an intention to treat basis. The remaining four trials were included in this review. They were published in full and contained data on 226 infants.

These studies were performed in Turkey, Germany, and Argentina. The dose of IVIG varied (table 1). Different IVIG preparations were used: Sandoglobulin $\left(\right.$ Sandoz) ${ }^{16}$; ISIVEN, Intravenous Immunoglobulin (Instituto Sierovaccinogeno Lucca, Italy) ${ }^{15}$; Polyglobin N (Cutter, Cologne, Germany). ${ }^{17}$ All studies stated that an attempt was made to administer the HDIVIG as soon as possible after the diagnosis of neonatal immune haemolytic disease was made and randomisation performed.

Infants with a history of prenatal treatment (maternal IVIG and/or in utero transfusion) were specifically excluded in one study. ${ }^{19}$ One study certainly included such infants and reported on number of in utero transfusions received and time since last one. ${ }^{16}$ It is unclear whether the other studies ${ }^{15}{ }^{17}$ did so.

Follow up and results data were complete in two studies. ${ }^{15} 16$ The methodological weakness of failing to report on an intention to treat basis for protocol violations was identified in one study; ${ }^{17}$ and another study ${ }^{19}$ reported results for 37 infants, having stated that 40 were eligible for inclusion in the study. From the latter study, it is unclear whether 37 or 40 infants were randomised at the beginning of the study. The criteria for phototherapy or exchange transfusion (table 1) varied between the studies. In one study ${ }^{16}$ phototherapy was started when the bilirubin level exceeded Oski and Naiman curves. ${ }^{21}$ In another, ${ }^{17}$ phototherapy was started when the bilirubin was less than $4 \mathrm{mg} / \mathrm{dl}$ below the exchange line on the Polácek graphs. ${ }^{22}$ It is not clear whether phototherapy was started immediately on entry into the study in the other studies.

\section{Outcomes measured}

Three studies $(n=189)$ reported on the number of exchange transfusions given. ${ }^{15-17}$ The fourth study ${ }^{19}$ reported exchange and red cell transfusions together. The results showed a significant reduction in the need for exchange transfusion after HDIVIG treatment (RR 0.28 (95\% CI 0.17 to 0.47 ), RD -0.37 (95\% CI -0.49 to -0.26$)$, number needed to treat (NNT) 2.7 (95\% CI 2.0 to 3.8$)$ ) (fig 1). There was no significant heterogeneity. Analysing the studies reporting this outcome 


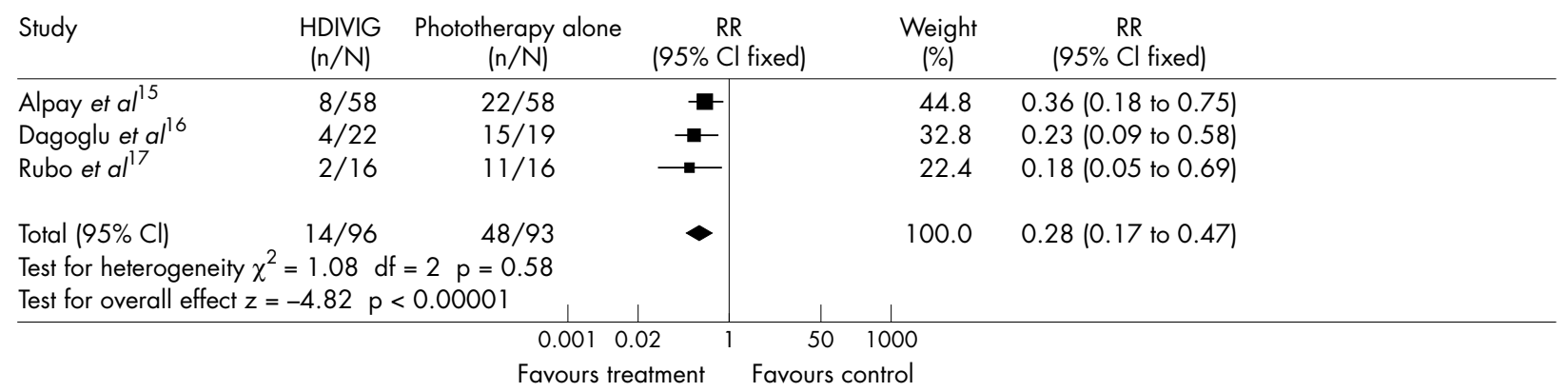

Figure 1 Number of infants requiring exchange transfusions when comparing high dose intravenous immunoglobulin (HDIVIG) with phototherapy alone.

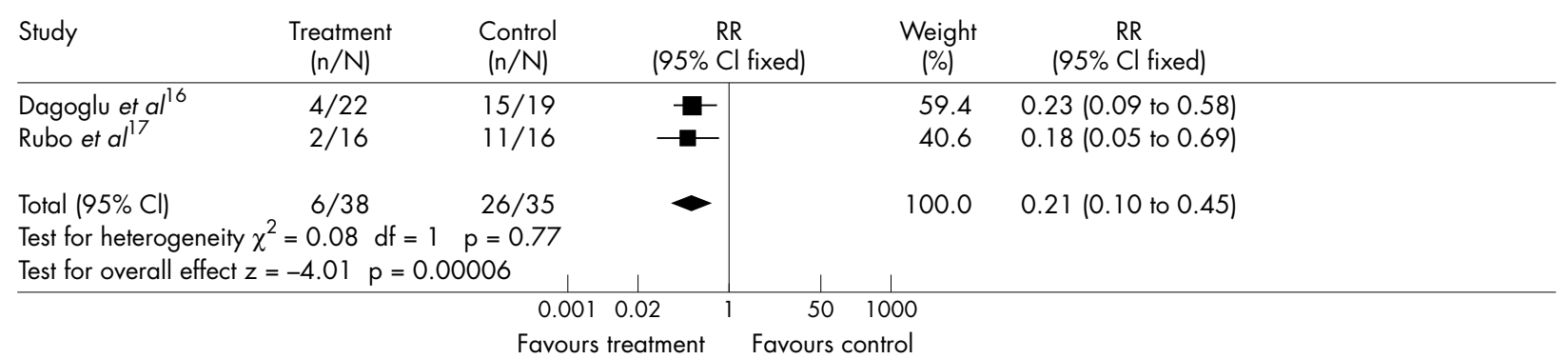

Figure 2 Number of infants requiring exchange transfusion when comparing high dose intravenous immunoglobulin with phototherapy alone (Rh disease only).

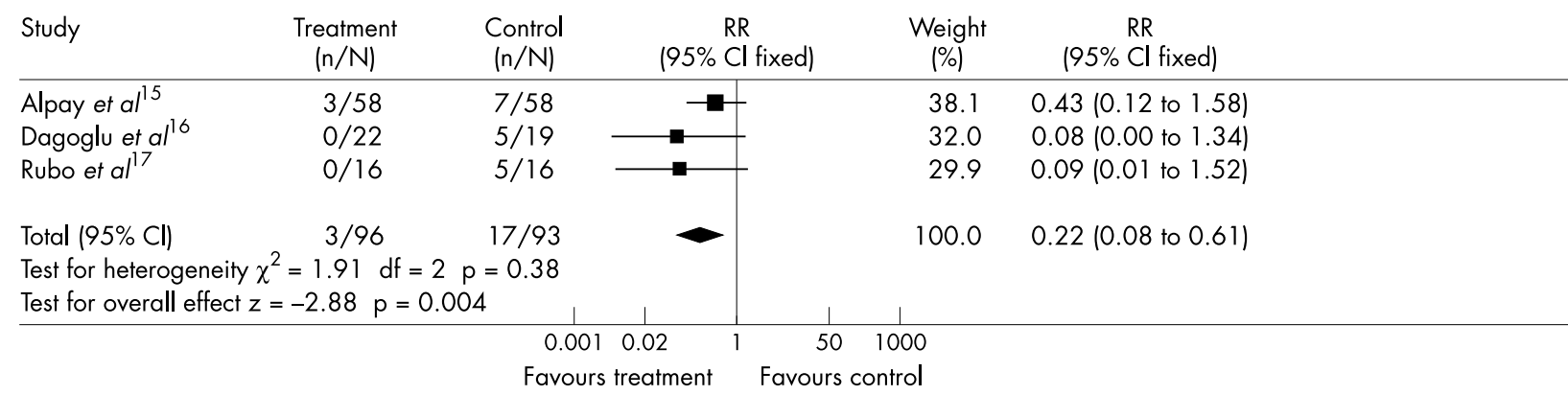

Figure 3 Number of infants requiring multiple exchange transfusions when comparing high dose intravenous immunoglobulin with phototherapy alone.

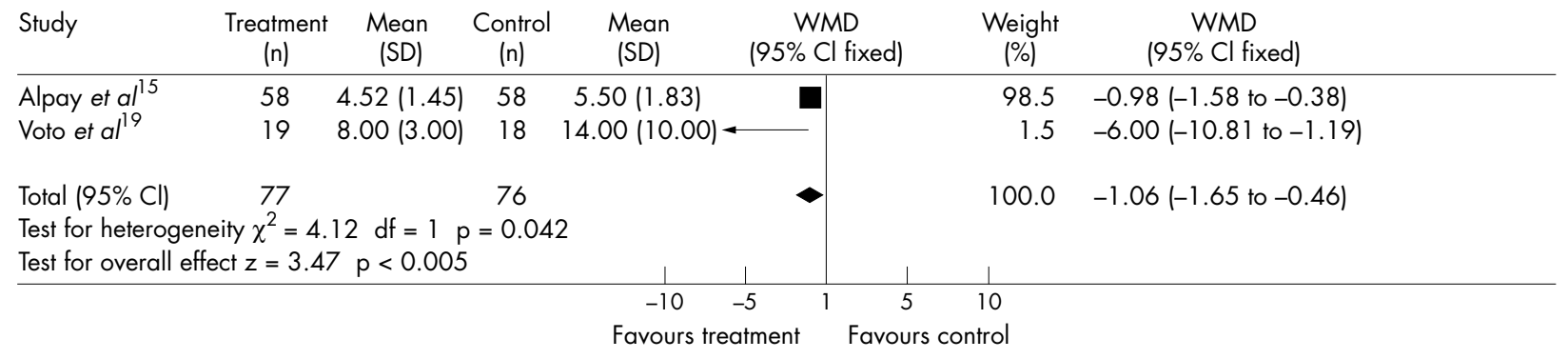

Figure 4 Mean length of hospital stay when comparing high dose intravenous immunoglobulin with phototherapy alone. WMD, Weighted mean difference.

for neonates with Rh haemolytic disease only $(\mathrm{n}=73),{ }^{16}{ }^{17}$ there is a further reduction in need for exchange transfusion (RR 0.21 (95\% CI 0.10 to 0.45 ), RD -0.59 (95\% CI -0.77 to -0.40 ), NNT 1.7 (95\% CI 1.3 to 2.5 )) (fig 2). There is also a significant reduction in the number of neonates requiring multiple exchange transfusions with HDIVIG treatment (RR 0.22 (95\% CI 0.08 to 0.61 ), RD -0.15 (95\% CI -0.24 to -0.06 )) (fig 3).

There is a significant reduction in the length of hospital stay with the use of HDIVIG treatment in addition to phototherapy
(WMD $-1.06(95 \%$ CI -1.65 to -0.46$)$ ) (fig 4). However, there is significant heterogeneity between the studies. Two studies $^{15}{ }^{19}$ reported on this outcome $(\mathrm{n}=153)$. Voto et al $^{19}$ found a considerably longer hospital stay.

There is a significant reduction in the duration of phototherapy required with HDIVIG treatment in addition to phototherapy (WMD -0.87 (95\% CI -1.37 to -0.37 )) (fig 5). Two studies ${ }^{15}{ }^{19}$ reported on this outcome $(n=153)$. Voto et $a l^{19}$ report data for this outcome; there was no difference in the two groups. Rübo et $\mathrm{al}^{17}$ reported that there was no difference 


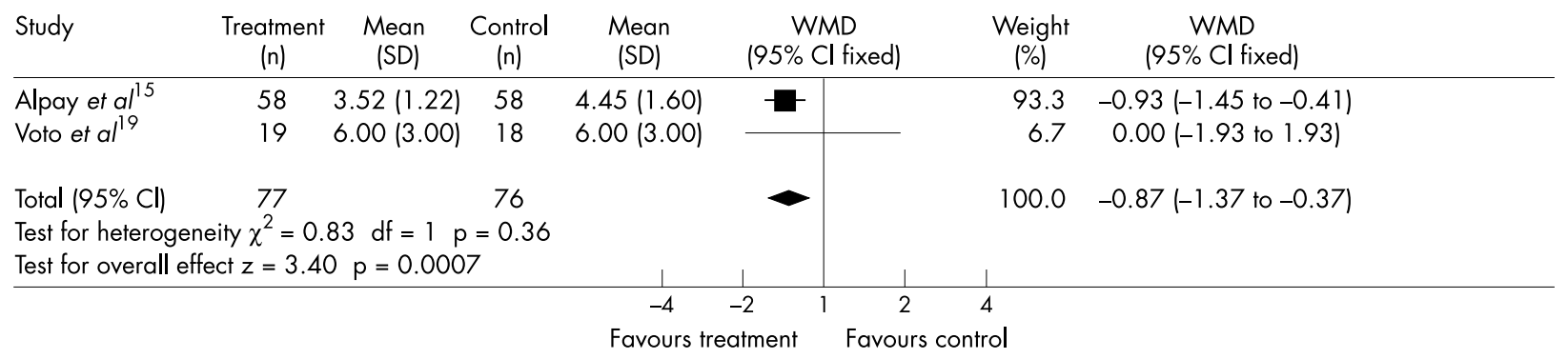

Figure 5 Mean duration of phototherapy when comparing high dose intravenous immunoglobulin with phototherapy alone. WMD, Weighted mean difference.

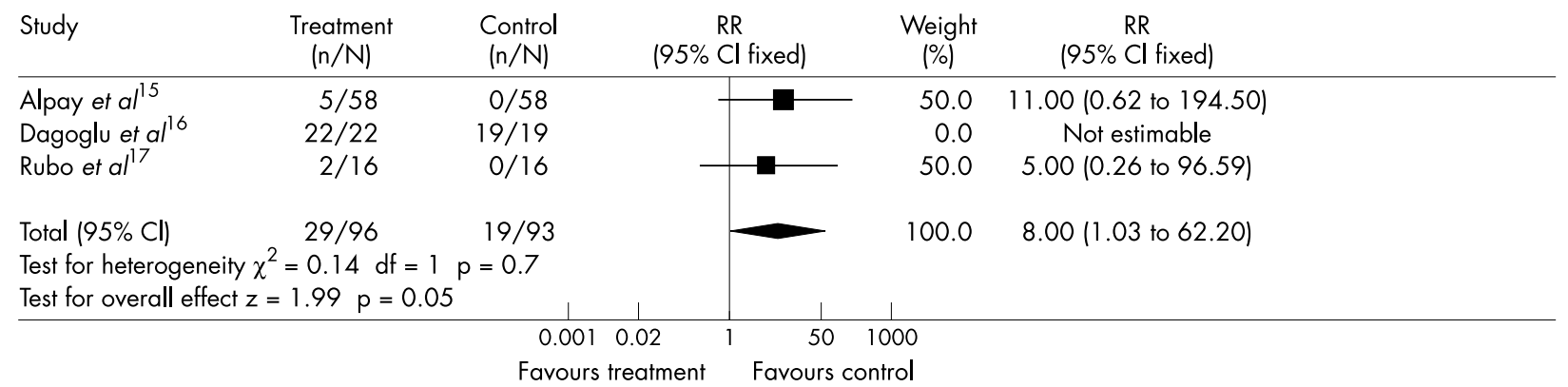

Figure 6 Number of infants requiring red cell transfusions for late anaemia when comparing high dose intravenous immunoglobulin with phototherapy alone.

between the two groups with respect to duration of phototherapy, although the data were not published.

There is an increase in the number of red cell transfusions required for late anaemia in those infants who received HDIVIG. This just reached significance with very wide confidence intervals (RR 8.0 (95\% CI 1.03 to 62.2), RD 0.07 (95\% CI 0.01 to 0.14$)$ ) (fig 6). Three studies $(\mathrm{n}=189)$ reported on this outcome. ${ }^{15-17}$ The criteria used in determining when these infants should be transfused were not always apparent.

\section{Reports of adverse events}

Three studies report that the infants were monitored closely for possible side effects of administration of HDIVIG ${ }^{.15-17}$ They observed infants for possible febrile, allergic, haemolytic, and volume overloading side effects of HDIVIG by observation of changes in body temperature, respiratory rate and pattern, heart rate, and blood pressure. None experienced any adverse events with the use of HDIVIG. Problems were, however, seen in infants of the control group who required exchange transfusions. Rübo et $a l^{17}$ report a case with inspissated bile syndrome and another with bacterial sepsis. A causal link was not established. Alpay et a ${ }^{15}$ report two cases in the control group who developed hypoglycaemia and hypocalcaemia after exchange transfusions.

\section{DISCUSSION}

Since the advent and widespread use of anti-D immunoglobulin prophylaxis in preventing sensitisation of Rh negative mothers, the incidence of $\mathrm{Rh}$ haemolytic disease of the newborn has fallen dramatically, although it still exists. In fact, anti-D remains the most common cause of morbidity and mortality from haemolytic disease, although its prevalence in relation to other red cell antibodies has appreciably declined. ${ }^{23}$ During a 12 month study of seven maternity units in Mersey and North Wales in 1993/1994, clinically important red cell antibodies were detected in $1 \%$ of the study population..$^{23}$ The morbidity and mortality associated with exchange transfusions are not insignificant. ${ }^{24}$ As the incidence of HDN has decreased in recent years, paediatricians are less experienced at performing exchange transfusions, and thus one might postulate that the morbidity associated with this procedure is likely to increase. To reduce the risks of exchange transfusions at the hands of inexperienced staff, the options for less invasive effective treatments such as HDIVIG should be explored.

The "number needed to treat" in order to prevent one exchange transfusion is very low at 2.7 (95\% CI 2.0 to 3.8 ). However, in these studies a large proportion of babies with Rh or $\mathrm{ABO}$ incompatibility in the control group required exchange transfusion despite phototherapy. The exchange transfusion rate in the controls is higher than may be expected in the United Kingdom. It is unclear why this is so. The exchange transfusion criteria used in the trials are fairly conventional. The difference may arise for a variety of reasons. Changes have occurred in the natural history of Rh disease with the use of anti-D and also in reproductive practice in Northern Europe. We can postulate that $(a)$ there are differences in antenatal antibody screening, $(b)$ direct Coomb's tests may only be performed on infants with very aggressive haemolysis, (c) phototherapy may have been inadequate, or $(d)$ there are other differences between the practices or populations in the United Kingdom and Turkey, Argentina, and Germany. Therefore it can be argued that the NNT in the United Kingdom would be higher than that reported in this systematic review. Nevertheless, HDIVIG is an effective treatment in reducing the exchange transfusion rate in neonates with HDN. Even in countries such as the United Kingdom where exchange transfusions are rarely performed in this clinical setting, the NNT would remain low if this treatment were reserved for infants with severe haemolysis, in whom the potential need for exchange transfusion is highest.

This review has shown a reduction in the duration of phototherapy and hospital stay when HDIVIG is used in addition to phototherapy for HDN. The financial saving from the shortened inpatient stay and duration of phototherapy offsets the cost of IVIG.

All the studies included showed a beneficial effect, despite the variation in IVIG doses. It appears that a single dose of 0.5 $\mathrm{g} / \mathrm{kg}$ on day $\mathrm{l}$ is as effective as any other treatment regimen. Some authors have implied that the key to successful 
treatment is the early administration of the immunoglobulin, as soon as the diagnosis of haemolytic anaemia is made.

When HDN is treated with HDIVIG in addition to phototherapy, there is a reduction in the degree of haemolysis and therefore the need for exchange transfusion. As the antibody has not been "washed out" (as would be the case during an exchange transfusion), there may be late haemolysis and anaemia. When the effect of the HDIVIG has worn off, the Fc sites on the surface of the reticuloendothelial cells become free to bind antibody sensitised neonatal erythrocytes, thus causing haemolysis. This is postulated to be the reason for the excess late red cell transfusions required in the HDIVIG treated group. The incidence of late anaemia may in fact be similar to that occurring in infants who had moderate haemolysis but did not require exchange transfusions.

At present, IVIG is licensed for use in primary immunodeficiencies and secondary immunodeficiencies such as chronic lymphocytic leukaemia, idiopathic thrombocytopenic purpura, Kawasaki disease, Guillain Barré syndrome, and paediatric AIDS. It is also used for intractable epilepsy and juvenile dermatomyositis. Although considered to be a relatively safe product, it is not without risk of adverse reactions. The identification of more than 100 patients with hepatitis $\mathrm{C}$ apparently acquired from a single immunoglobulin product is a reminder that IVIG is a biological product derived from pooled human plasma. ${ }^{25}$ To reduce the risk of transmission of infective agents, donors and donations are selected and plasma pools are tested and undergo a viral partitioning and inactivation process. ${ }^{26}$ However, the small risk of potential transfusion transmitted diseases will remain.

Other adverse effects reported from the use of IVIG include headache, meningism, fever, nausea, low back pain, transient hypotension, and, rarely, a mild, usually clinically irrelevant, haemolytic anaemia. ${ }^{27}$ These adverse effects are usually mild and transient. Serious side effects are very rare and include hypersensitivity and anaphylactic reactions, which can occur in patients with IgA deficiency or hypogammaglobulinaemia as a result of the presence of IgA antibodies in the patient's serum. ${ }^{28}$

\section{Implications for future research}

The evidence presented in this systematic review of the available randomised controlled trials of HDIVIG administered in the postnatal period in HDN shows a significant effect for HDIVIG. Thus, it may be considered unethical to delay wider use of HDIVIG while carrying out further research. The optimum treatment dose and frequency is as yet uncertain and this may be an area for further research. Multiple doses of IVIG may reduce the late anaemia and therefore reduce the need for red cell transfusions. In the study investigating the role of repeated doses of HDIVIG, there appears to be a reduction in the need for exchange transfusion in the group receiving a second dose of IVIG 48 hours after the first. ${ }^{20}$ However, the numbers are small ( 25 in each study arm), and flaws occurred in the method of randomisation.

\section{Authors' affiliations}

R Gottstein, R W I Cooke, Neonatal Unit, Liverpool Women's Hospital, Liverpool, UK

\section{REFERENCES}

1 Imbach P, d'Apuzzo V, Hirt A, et al. High dose intravenous gammaglobulin for idiopathic thrombocytopenic purpura in childhood. Lancet 1981;1:1228-31.
2 Bolton-Maggs PHB, Moon I. Assessment of UK practice for management of acute childhood idiopathic thrombocytopenic purpura against published guidelines. Lancet 1997;350:620-3.

3 Fehr J, Hofmann V, Kappeler U. Transient reversal of thrombocytopenia in idiopathic thrombocytopenic purpura by high-dose intravenous gamma globulin. N Engl J Med 1982;306:1254-18.

4 Wenkse G, Gaedicke G, Heyes H. Idiopathic thrombocytopaenic purpura in pregnancy and neonatal period. Blut 1984:48:377-82.

5 Bussel JB, Berkowitz RL, Lynch L, et al. Antenatal management of alloimmune thrombocytopenia with intravenous $\gamma$-globulin: a randomized trial of the addition of low-dose steroid to intravenous $\gamma$-globulin. Am J Obstet Gynecol 1996;174:1414-23.

6 Voto LS, Mathet ER, Zapaterio JL, et al. High-dose gammaglobulin (IVIG) followed by intrauterine transfusions (IUTs): a new alternative for the treatment of severe fetal hemolytic disease. J Perinat Med 1997;25:85-8.

7 Doreen MC, van-Kamp IL, Scherpenisse JW, et al. No beneficial effect of low-dose fetal intravenous gammaglobulin administration in combination with intravascular transfusions in severe Rh $D$ haemolytic disease. Vox-Sang 1994;66:253-7.

8 Alonso JG, Decaro J, Marrero A, et al. Repeated direct fetal intravascular high-dose immunoglobulin therapy for the treatment of $\mathrm{Rh}$ hemolytic disease. J Perinat Med1994:22:415-19.

9 Ouwehand WH, Smith G, Ranasinghe E. Management of severe alloimmune thrombocytopenia in the newborn. Arch Dis Child Fetal Neonatal Ed 2000;82:F173-5.

10 Ohlsson A, Lacy JB. Intravenous immunoglobulin for preventing infection in preterm and/or low-birth-weight infants. (Cochrane Review). The Cochrane Library Oxford: Update Software, 2000:issue 3.

11 Stanley TV, Grimwood K. Classical Kawasaki disease in a neonate. Arch Dis Child Fetal Neonatal Ed 2002;86:F135-6.

12 Chitkara U, Bussel J, Alvarez M, et al. High-dose intravenous gamma globulin: does it have a role in the treatment of severe erythroblastois fetalis? Obstet Gynecol 1990:76:703-8.

13 Urbaniak SJ. ADCC (K cell) lysis of human erythrocytes sensitized with Rhesus alloantibodies. II. Investigation into mechanism of lysis. Br J Haematol 1979;42:315-28.

14 Jadad AR, Rennie D. The randomised controlled trial gets a middle-aged checkup. JAMA 1998;279:319-20.

15 Alpay F, Sarici SÜ, Okutan V, et al. High-dose intravenous immunoglobulin therapy in neonatal immune haemolytic jaundice. Acta Paediatr 1999:88:216-19.

16 Dagoglu T, Ovali F, Samaci N, et al. High-dose intravenous immunoglobulin therapy for rhesus haemolytic disease. J Int Med Res 1995;23:264-71.

17 Rübo J, Albrecht K, Lasch $\mathrm{P}$, et al. High-dose intravenous immune globulin therapy for hyperbilirubinemia caused by Rh hemolytic disease. J Pediatr 1992;121:93-7.

18 Rübo J, Wahn V. Hochdosierte Immunglobulintherapie der Hyperbilirubinämie bei Rhesusinkompatibilität. Infusionsther Transfusionsmed 1993;20:104-9.

19 Voto LS, Sexer H, Ferreiro G, et al. Neonatal administration of high-dose intravenous immunoglobulin in rhesus hemolytic disease. J Perinat Med 1995:23:443-51.

20 Rübo J, Wahn V, et al. Influence of high dosage immuno-globulin therapy on hyperbilirubinemia in rhesus hemolytic disease. A co-operative study. Monatsschr Kinderheilk 1996;144:516-19.

21 Oski FA, Naiman JL. Erythroblastosis fetalis. In: Oski FA, Naiman JL, eds. Haematological problems in the newborn. Philadelphia: WB Saunders Company, 1982:283-346.

22 Polácek K. Das universale Diagramm zur Behandlung der Hyperbilirubinämie der Neugeborenen. Pädiatrische Praxis 1984;29:1-3

23 Howard H, Martlew V, McFayden I, et al. Consequences for fetus and neonate of maternal red cell allo-immunisation. Arch Dis Child Fetal Neonatal Ed 1998;78:F62-6.

24 Keenan WJ, Kazmaier Novak K, Sutherland JM, et al. Morbidity and mortality associated with exchange transfusion. Pediatrics 1985;75:417-21

25 Healey CJ, Sabharwal NK, Daub J, et al. Outbreak of acute hepatitis C following the use of anti-hepatitis $C$ virus-screened intravenous immunoglobulin therapy. Gastroenterology 1996;110:1120-6.

26 Yap PL. Intravenous immunoglobulin and hepatitis $C$ virus: an overview of transmission episodes with emphasis on manufacturing data. Clin Ther 1996;18(suppl B):43-58.

27 Dunhem C, Dicato MA, Ries F. Side-effect of intravenous immune globulin. Clin Exp Immunol 1994;97(suppl 1):79-83.

28 Burks AW, Sampson HA, Buckley RH. Anaphylactic reactions after gammagloblin administration in patients with hypogammaglobulinaemia. Detection of lgE antibodies to lgA. N Engl J Med 1986;314:560-3. 